ISAHP Article: Arbel, Vargas/Weighted Euclidean Centers and Interval Receiprocal Matrices Submitted to the International Symposium of the Analytic Hierarchy Process 2014, Washington D.C., U.S.A.

\title{
WEIGHTED EUCLIDEAN CENTERS AND INTERVAL RECIPROCAL MATRICES
}

\author{
Ami Arbel \\ School of Engineering \\ Tel Aviv University \\ Tel Aviv, Israel \\ E-mail: ami@eng.tau.ac.il \\ Luis G. Vargas \\ The Joseph M. Katz Graduate School of Business \\ University of Pittsburgh \\ Pittsburgh, PA, USA \\ E-mail: lgvargas@pitt.edu
}

\begin{abstract}
This paper addresses the derivation of a Euclidean center and its application to interval reciprocal matrices. An Euclidean center is defined as the point in decision space from which one can inscribe the largest sphere contained by the constraints. We extend this concept by introducing its weighted version, which we term the weighted Euclidean center. We show that by assigning weights to the different decision variables we can traverse the entire decision space. In addition, we show that the concept of a weighted Euclidean center and that of the achievement scalarizing function introduced by Wierzbicki are intimately related.
\end{abstract}

Keywords: Interval judgments, reciprocal matrices, weighted Euclidean center. 